\title{
Dimensions of Polystyrene Particles Deposited on Mica from Dilute Cyclohexane Solution at Different Temperatures
}

\author{
Mustafa M. Demir ${ }^{\dagger}$ and Burak Erman*,t, \\ Faculty of Engineering and Natural Sciences, Sabanci University, Orhanli, \\ 81474 Tuzla, Istanbul, Turkey, and Departments of Chemistry and Mechanical Enginering, \\ Koc University, Rumelifeneri Yolu, Sariyer 80910, Istanbul, Turkey \\ Received May 2, 2002; Revised Manuscript Received J uly 25, 2002
}

\begin{abstract}
Using atomic force microscopy, the height, diameter, and volume of polystyrene particles deposited on mica from dilute cyd ohexane solution at different temperatures are determined. Dimensions exhibit a strong temperature dependence. The heights of the deposited particles are only a few atomic diameters, the major dimension being parallel to the mica surface. The number of single polystyrene molecules in a deposited particle cannot be determined directly by atomic force microscopy. However, the maximum number of molecules that may be present in a particle may be estimated. Bel ow $35^{\circ} \mathrm{C}$, the particles possibly consist of single collapsed molecules. This number increases with temperature and becomes as large as about 30 molecules per particle at $80^{\circ} \mathrm{C}$. The volume occupied by a single chain in solvent is calculated as a function of temperature and compared with observed volumes of dry particles on mica. A linear relationship is observed between single chain volumes in solvent and corresponding particle vol umes on mica. On the average, the vol ume of a single chain in solution is 10 times the volume of a dry particle at the corresponding temperature. Fluctuations of chain vol umes that are dominant in solution are also present in the volumes of particles deposited and dried on mica.
\end{abstract}

\section{Introduction}

Dimensions of a single polymer chain in solvent at different temperatures depend on the structural features of the chain and the interaction between the chain and the solvent. The temperature dependence of the interaction is most significant for the system polystyrene (PS)-cyclohexane ( $\mathrm{CH})$, which has been studied extensively in the past, both experimentally and theoretically. ${ }^{1-7}$ A single PS chain in $\mathrm{CH}$ exhibits a rapid decrease of dimensions when the temperature is decreased below about $35{ }^{\circ} \mathrm{C}$. This phenomenon is known as the coil-globule transition. For sufficiently long PS chains, it has been shown, by using thermodynamic and statistical mechanical arguments, that the system PS-CH goes through a critical transition, $1,8,10$ which is the reason for the observed abrupt change in the dimensions of PS at the stated temperature. In systems that do not go through or near critical conditions as the solvent quality becomes poorer, the dimensions of the chains also decrease. However, this change takes place gradually, and a rapid "collapse-like" transition or a sharp coil-globule transition is not possible.

The system PS- $\mathrm{CH}$ is representative in this respect. The observation of large dimensional changes in single chains at easily accessible temperatures is especially important for technological applications at the molecular scale. In the present study, we observe, by an atomic force microscope (AFM), the dimensions of the PS particles deposited and dried on mica surfaces from dilute cyclohexane solution at temperatures above, at, and below the $\Theta$ temperature. A strong temperature dependence of the dimensions is observed. Unfortunately, however, AFM measurements cannot indicate whether the deposited particles consist of single or several chains. Here, we interpret the results of mea-

\footnotetext{
† Sabanci University.

₹ Koc University.
}

surements in terms of two different scenarios. The first scenario is based on the single chain per particle picture. This is based on the fact that the solutions from which the particles are deposited are extremely dilute and on the assumption that individual molecules remain separated during evaporation. The single chain per particle assumption leads to the cal culation of the deformations that take place in the chains during evaporation. The second scenario is based on the several chains per particle picture. In this picture, the individual chains in solution aggregate during evaporation. The maximum possible number of chains per particle can be estimated because the upper bound of the segment density of the molecules on the surface is the bulk density. If the observed particles have bulk densities, then the number of particles in each, i.e., the maximum possible number, may be calculated by dividing its vol ume by the vol ume of a single collapsed chain. This number turns out to be strongly dependent on temperature. Irrespective of the number of chains per particle, we see that the distribution of particle sizes on mica at a given temperature is related in a simple way to the calculated distribution of chain dimensions in solution at the same temperature.

Single PS molecules deposited on mica or silicon from various solutions have previously been investigated by Qian et al.,11 Festag et al.,12 Stange et al.,13 and Zhang et al. ${ }^{14}$ using STM, AFM, and electron microscopy. Qian et al. ${ }^{11}$ investigated the morphology of electrosprayed PS particles from solvents of different quality and the changes in morphology over long times of observation. Zhang et al. ${ }^{11}$ used the droplet evaporation technique on a graphite surface and identified the dimensions of different molecular weight PS molecules. Stange et al. ${ }^{13}$ studied the effects of concentration and molecular weight on discontinuous PS films on silicon surfaces. In this paper, we present a systematic AFM analysis of the temperature dependence of dimensions of PS molecules cast from $\mathrm{CH}$. 
In section 2 we describe the experimental procedure for depositing and observing the PS molecules on mica surfaces. In section 3 we report results of experiments, followed by a theoretical interpretation of the results in section 4 .

\section{Experimental Section}

Materials. PS $\left(2 \times 10^{6} \mathrm{~g} / \mathrm{mol}\right.$ molecular weight, $\mathrm{M}_{\mathrm{w}} / \mathrm{M}_{\mathrm{n}}=$ 1.30) was provided from Alfa Aesar (lot no. K29J 21). Cyclohexane was obtained from Reidel-de Haen and used as received. A dilute solution of PS in $\mathrm{CH}, 4.6 \times 10^{-6} \mathrm{~g} / \mathrm{mL}$, was prepared at room temperature. Concentrations lower and higher than $4.6 \times 10^{-6} \mathrm{~g} / \mathrm{mL}$ were also investigated. However, the number of particles deposited on mica was too few to allow for a rigorous analysis at lower concentrations, and aggregation effects were observed at higher concentrations..$^{2,12}$ The optimum concentration of $4.6 \times 10^{-6} \mathrm{~g} / \mathrm{mL}$ was therefore used throughout all measurements.

Sample Preparation. All specimens were prepared by depositing a drop of the solution onto freshly cleaved mica surfaces. ${ }^{14}$ The top layer of mica approximately $1 \mathrm{~cm}^{2}$ was peel ed by using Scotch tape to make a fresh, clean, and flat surface. Equal volumes of polymer solution approximately 40 $\mu \mathrm{L}$ were deposited on the freshly cleaved mica by a micropipet. The deposition was carried out immediately following the cleavage to avoid oxidation of the mica surface. The solutions were deposited on mica at $25,35,50$, and $80^{\circ} \mathrm{C}$. The mica substrate and polymer solution were left to dry in an oven for $24 \mathrm{~h}$ at the temperature of deposition. The samples were then transferred to a desiccator under low vacuum. Humidity was controlled and kept under $40 \%$ inside the desiccator. All samples were prepared at least three times. The highest temperature, $80^{\circ} \mathrm{C}$, is close to the boiling temperature of $\mathrm{CH}$. To investigate possible anomalous effects at such a high temperature, several experiments were al so carried out around $70{ }^{\circ} \mathrm{C}$. No anomalous results were observed.

AFM I maging. Tapping mode in air AFM (Nanoscope IIIa, Digital Instruments) imaging was carried out with oxide sharpened Si tips. Dimensi ons of the adsorbed molecules were measured at room temperature by using bearing analysis and section analysis options of the Nanoscope software. In addition to humidity control, the head of the AFM was flushed with liquid $\mathrm{N}_{2}$ before start of the measurement. ${ }^{15} \mathrm{AFM}$ images are recorded in the height and phase modes without any image processing except flattening and plane fitting. Comparing at least two images of different samples verified the reproducibility of the images. More than 230 data were collected from three different reproducible images for each temperature value. At least 50 particles were examined for obtaining height and diameter distributions for each temperature. Several freshly cleaved mica surfaces, as well as mica surfaces on which pure cyclohexane was deposited and evaporated, were studied under the AFM in order to distinguish between PS particles and possible impurities on the surface. The freshly cleaved mica showed no structure within the length scales of our observations $(0.5-500 \mathrm{~nm})$. On surfaces onto which pure $\mathrm{CH}$ was deposited and evaporated, we observed occasional spherical particles. However, the number of these particles was very small, and their di mensions were approximately an order of magnitude smaller than the smallest PS particles observed at $25^{\circ} \mathrm{C}$

Two sources of uncertainty may be affecting the observations. First, particles observed with microscope may contain cyclohexane bound to PS although the samples remain in the oven for $24 \mathrm{~h}$. Second, the dimensions of particles may be somewhat overestimated by AFM due to tip geometry, known as tip convolution. To obtain actual diameter of particles, the radius of the tip and the particle should betaken into account. The corrected particle radius is estimated by Festag et al.,12 assuming the tip and the particle as hemispheres, according to the expression

$$
r_{\text {particle }}=\left[r_{\text {tip }}^{2}+r_{\text {observed }}^{2}\right]^{1 / 2}-r_{\text {tip }}
$$
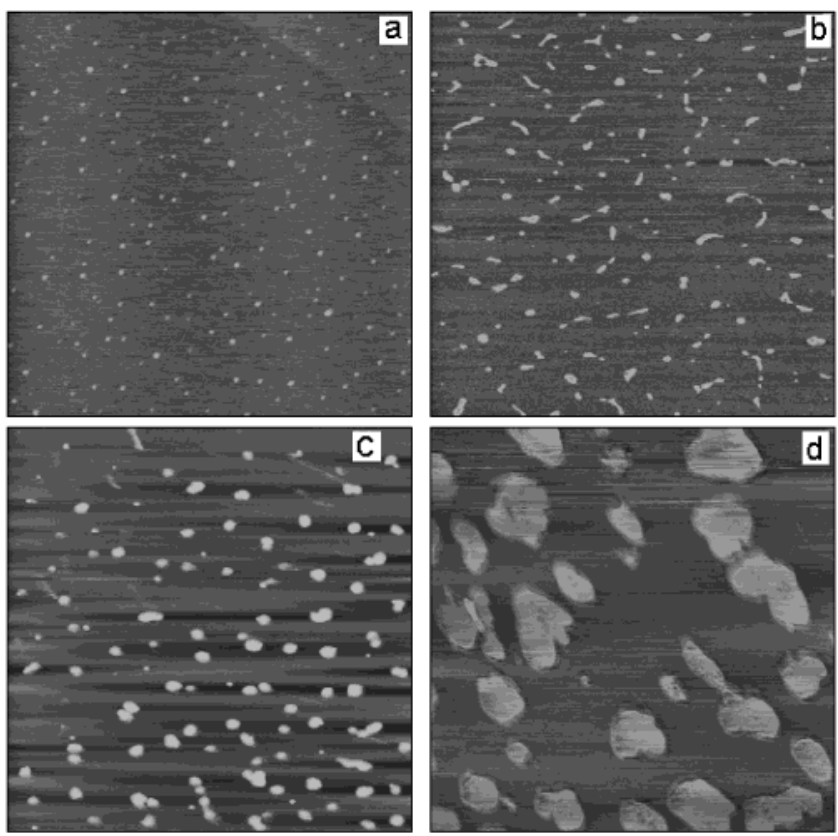

Figure 1. AFM images of polystyrene particles deposited from a very dilute solution of PS in CH onto a mica substrate at (a) 25 , (b) 35 , (c) 50 , and (d) $80{ }^{\circ} \mathrm{C}$. All images have $5 \times 5 \mu \mathrm{m}^{2}$ scan area.

The second factor is described in some detail in ref 11. It should al so be noted that whether an observed particle consists of a single chain or more cannot be directly inferred from the AFM measurements. To understand whether the observed particles consist of single chains, observations were also made on particles obtained from solutions with concentrations of $10^{-8}$ $\mathrm{g} / \mathrm{mL}$, at which the particles are expected to consist of single chains. These measurements indicated that the dimensions of the partides observed at extremely low concentrations agree with a statistically significant portion of the distribution of sizes observed at higher concentrations.

\section{Results of Measurements}

Four AFM images of PS molecules on mica surfaces are shown in Figure $1 \mathrm{a}-\mathrm{d}$, obtained at four temperatures, $25,35,50$, and $80^{\circ} \mathrm{C}$. The shapes of the particles are round and symmetric except those obtained at 35 ${ }^{\circ} \mathrm{C}$. As the temperature increases, the dimensions (height, diameter, and volume) of the particles increase, and their number density per unit area decreases. For example, there are 172 particles $/ 25 \mu \mathrm{m}^{2}$ in the image of $25^{\circ} \mathrm{C}$ whereas the second, third, and fourth images have 149,102 , and 28 , respectively. All of the particles are well separated from each other as can be seen from the AFM images of Figure 1.

A drop of polymer solution, approximately $10 \mu \mathrm{L}$ in volume and with a concentration of $10^{-8} \mathrm{~g} / \mathrm{mL}$, spreads out in the form of a circular region when deposited on mica. The diameter of the circular region diminishes gradually as the solvent evaporates. After complete evaporation of the solvent, areas of $4 \mu \mathrm{m}^{2}$ were scanned in the region initially occupied by the drop. Resulting images show that the polymer molecules move toward the perimeter of the drop as the solvent evaporates. The number of polymer molecules per unit area was markedly less at the center of the drop than at the perimeter, as can be seen from Figure 2. This observation is in agreement with the recent theoretical predictions of Deegan et al. ${ }^{16}$

The dimensions of the particles were examined as height, diameter, and volume. To determine the diam- 

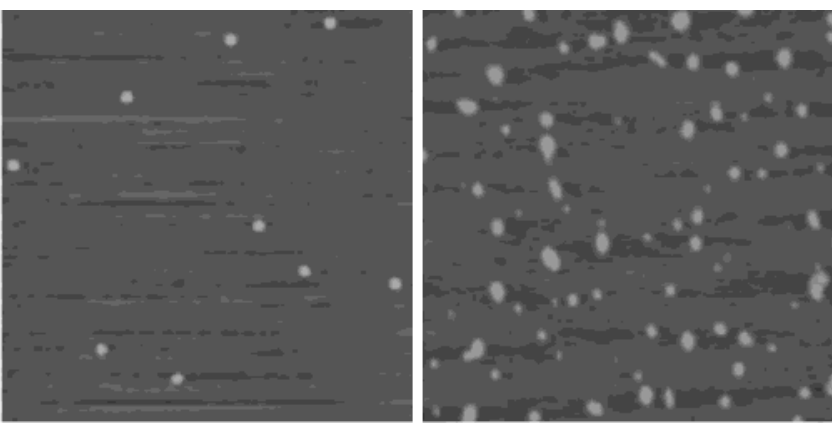

Figure 2. AFM height images captured from (a) center and (b) perimeter of the drop after solvent evaporation. I mages have $2 \times 2 \mu \mathrm{m}^{2}$ scan area.

Table 1. Experimentally Measured Particle Dimensions on Mica at Four Different Temperatures

\begin{tabular}{|c|c|c|c|c|}
\hline $\mathrm{T},{ }^{\circ} \mathrm{C}$ & $\begin{array}{c}\text { vol } \\
\text { (dry, on mica), } \\
\mathrm{nm}^{3}\end{array}$ & $\begin{array}{c}\text { diameter } \\
\text { (measd), } \\
\mathrm{nm}\end{array}$ & $\begin{array}{c}\text { diameter } \\
\text { (corrected), } \\
n m\end{array}$ & $\begin{array}{c}\text { height } \\
\text { (dry, on mica), } \\
\mathrm{nm}\end{array}$ \\
\hline $\begin{array}{l}25 \\
35 \\
50 \\
80\end{array}$ & $\begin{array}{r}4000 \\
11000 \\
50000 \\
125000\end{array}$ & $\begin{array}{r}65 \\
70 \\
136 \\
506\end{array}$ & $\begin{array}{r}52 \\
57 \\
122 \\
491\end{array}$ & $\begin{array}{l}1.0-1.5 \\
2.5-3.0 \\
4.0-4.5 \\
0.5-1.0\end{array}$ \\
\hline
\end{tabular}

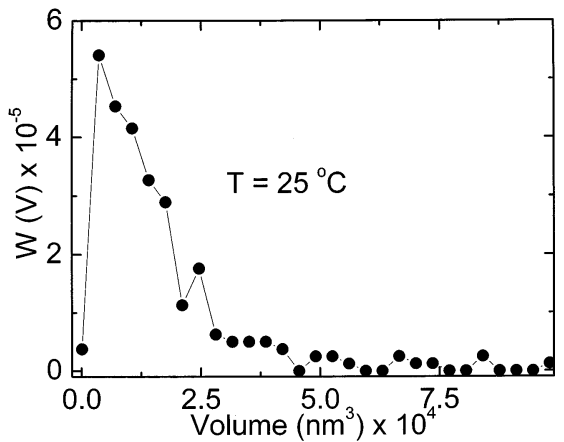

Figure 3. Volume distribution of polystyrene particles at 25 ${ }^{\circ} \mathrm{C}$. The bin size is $3500 \mathrm{~nm}^{3}$.

eter, the principal axes of the particles were determined in a 2-dimensional view. The major and the minor horizontal distances, $d_{1}$ and $d_{2}$, respectively, were measured for each particle, and the diameter $d$ was eval uated according to the expression $d=\left[d_{1} d_{2}\right]^{1 / 2}$. The height of each particle was taken as the vertical distance between the plane of the mica and the highest point of the protrusion above the smooth plane of mica. The volume of the particle above the smooth mica plane was determined using the bearing volume analysis options of Nanoscope III software. Results of measurements of the PS particles at four different temperatures are presented in the second, third, and fifth columns of Table 1. The diameter values in the fourth col umn are corrected according to eq 1 . The height values in the fifth column are given in the form of a range of values owing to the uncertainties resulting from the smallness of the height values.

The distribution of the measured volumes is presented in Figures 3-6 for the four temperatures. The filled circles represent experimental data, and the curves through the points are drawn to guide the eye. The ordinate represents the distribution of the observed volumes, normalized to unity. A striking feature of the curves is that the volumes are distributed over a wide range of values at all temperatures and that all of the distributions are strongly skewed, with a long tail toward larger values of the volume.

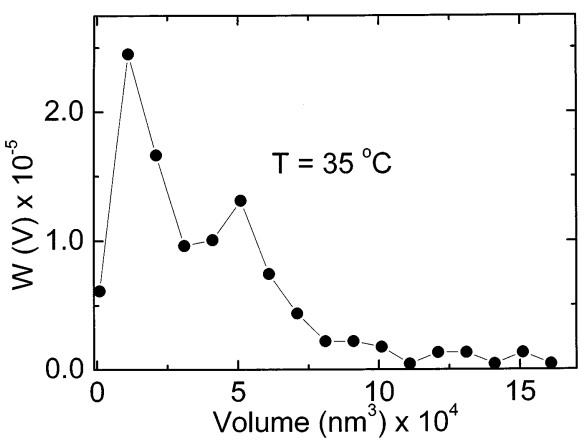

Figure 4. Volume distribution of polystyrene particles at 35 ${ }^{\circ} \mathrm{C}$. The bin size is $10000 \mathrm{~nm}^{3}$.

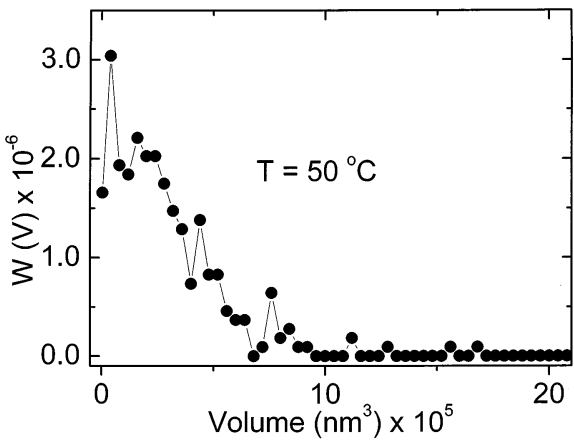

Figure 5. Volume distribution of polystyrene particles at 50 ${ }^{\circ} \mathrm{C}$. The bin size is $40000 \mathrm{~nm}^{3}$.

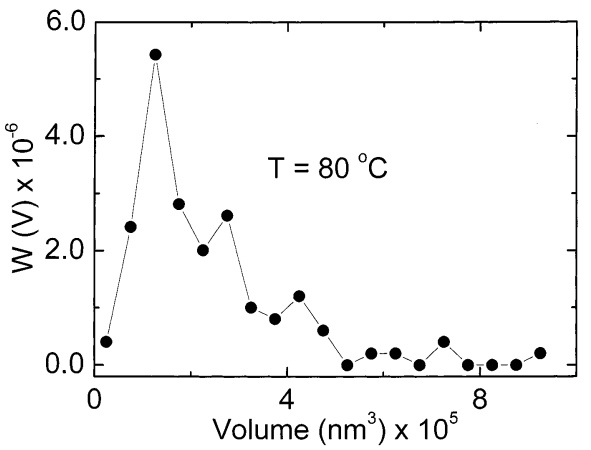

Figure 6. Volume distribution of polystyrene particles at 80 ${ }^{\circ} \mathrm{C}$. The bin size is $50000 \mathrm{~nm}^{3}$.

The volume of the single gl obular polymer molecule can be calculated from its bulk density $\left(1.05 \mathrm{~g} / \mathrm{cm}^{3}\right)^{12,14}$ For the molecular weight of $2 \times 10^{6} \mathrm{~g} / \mathrm{mol}$, this volume is $3200 \mathrm{~nm}^{3}$. This value is close to the peak value of the distribution in Figure 3. Considering that the chains are al ready in the globular state in solution at $25^{\circ} \mathrm{C}$, we may conclude that their deposition and subsequent drying does not further affect their dimensions. The larger volumes observed in the distribution of Figure 3 may be mainly due to two reasons. Either there may be more than one chain in the larger particles observed or the particles may consist of single chains which were not gl obular in the $\mathrm{CH}$ solution and remained nonglobular during drying. AFM measurements cannot differentiate between these two cases. Similar arguments apply to chains deposited at higher temperatures, shown in Figures 4-6.

To further elaborate on the effects of the numbers of chains in a particle, we study the problem in terms of two limiting scenarios:

(1) The Single Chain per Particle Scenario. The concentrations of solutions used in the present study 
Table 2. Calculated Dimensions of Single PS Chains in $\mathrm{CH}$ at Four Different Temperatures

\begin{tabular}{|c|c|c|c|c|}
\hline $\mathrm{T},{ }^{\circ} \mathrm{C}$ & $\begin{array}{c}\text { vol } \\
\text { (solution), } \\
\mathrm{nm}^{3} \text {, calcd }\end{array}$ & $\begin{array}{l}\text { diameter } \\
\text { (solution), } \\
\mathrm{nm} \text {, calcd }\end{array}$ & $\lambda_{\|}$ & $\lambda_{\perp}{ }^{a}$ \\
\hline 25 & 32000 & 40 & 1.30 & 0.031 \\
\hline 35 & 210000 & 74 & 0.77 & 0.037 \\
\hline 50 & 600000 & 105 & 1.16 & 0.040 \\
\hline 80 & 1200000 & 132 & 3.72 & 0.006 \\
\hline
\end{tabular}

a The averages of the height values of column 5 of Table 1 are used in obtaining this ratio.

Table 3. Maximum Possible Number of Chains in a Particle on Mica at Four Different Temperatures

\begin{tabular}{ccc}
\hline $\mathrm{T},{ }^{\circ} \mathrm{C}$ & $\begin{array}{c}\text { obsd dry vol, } \\
\mathrm{nm}^{3}\end{array}$ & $\begin{array}{c}\text { no. of chains } \\
\text { per particle }\end{array}$ \\
\hline 25 & 4000 & 1 \\
35 & 11000 & 3 \\
50 & 50000 & 13 \\
80 & 125000 & 32
\end{tabular}

are sufficiently low to ensure that the individual chains in the solution are well separated from each other. If the chains also remain well separated during deposition on the mica surface and during subsequent evaporation, then the observed particles should contain single PS molecules each. In this case the segmental density in the measured volume is low which is essentially an envel ope encl osing the total space needed to contain the chain. The volume of a single chain in solution is calculated, as described in the next section and presented in the second column of Table 2. The corresponding diameters, taking the chain to be spherical, are presented in column 3. At the beginning of deposition, the chains pervade a spherical volume. During evaporation, however, they are compressed strongly in the direction perpendicular to the mica surface and are expanded laterally parallel to the surface. The expansion ratio, $\lambda_{\|}$, defined as the ratio of the observed diameter on mica to the diameter in solution is presented in the fourth column of Table 2. The compression ratio, $\lambda_{\perp}$, defined as the ratio of the observed height to the diameter in solution is presented in the fifth column of Table 2 . On the average, the decrease in volume in going from solution to the dry state is approximately 10-fold.

(2) Several Chains per Particle Scenario. If the attractive intermolecular forces during evaporation are dominant, several single chains may adhere together to form a particle having clusters of single chains. In the extreme case that the clusters formed in this manner have segmental densities equating to the bulk density, then the number of molecules in each cluster may be estimated as the ratio of the observed volume to the volume of a single gl obular chain. In Table 3, the maximum number of chains per particle, calculated in this way by taking the volume of a single chain as 4000 $\mathrm{nm}^{3}$, is presented as a function of temperature. The third column of the table shows that the number of molecules in a particle increases strongly with temperature.

The width of the distributions in Figures 3-6 depends strongly on temperature as may be verified from a comparison of abscissa values.

The distributions of diameters are presented in Figure 7 for the four temperatures. The points represent experimental data, and the lines are drawn to guide the

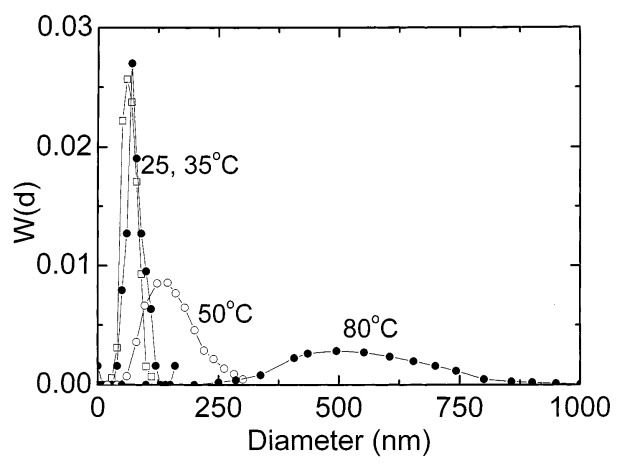

Figure 7. Diameter distribution of polystyrene particles at $25,35,50$, and $80^{\circ} \mathrm{C}$.

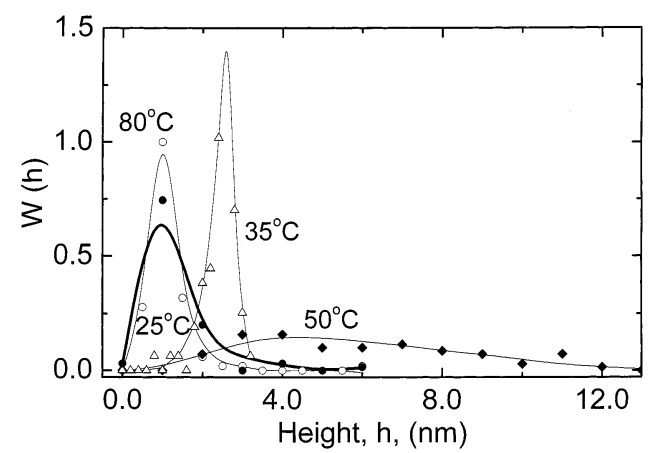

Figure 8. Height distribution of polystyrene particles at 25 , 35,50 , and $80{ }^{\circ} \mathrm{C}$.

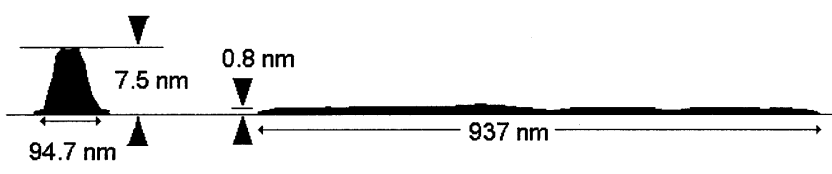

(a)

(b)

Figure 9. Section analysis of two different morphologies of PS: (a) round and (b) flat.

eye. The distributions for 25 and $35{ }^{\circ} \mathrm{C}$ are sharply peaked and are approximately the same. The distributions for 50 and $80{ }^{\circ} \mathrm{C}$ are wider, the latter showing a significant degree of dispersion about the mean value.

The distributions of heights are presented in Figure 8. The points represent experimental data, and the curves are drawn to guide the eye. The curve through the points for $\mathrm{T}=25^{\circ} \mathrm{C}$ is drawn heavier in order to differentiate it from the curve for $80^{\circ} \mathrm{C}$. The height values at $80{ }^{\circ} \mathrm{C}$ are low due to extensive spreading of the molecules on the mica surface. Otherwise, the features of the height distributions parallel those for diameter distributions, as evident from the comparison of Figures 7 and 8.

Morphology of PS Structures: Round vs Flat. Above $35{ }^{\circ} \mathrm{C}$, the temperature of the substrate on which the solution is deposited strongly affects the shape of the particles. If, during deposition, the mica is at room temperature and the solution is at $50{ }^{\circ} \mathrm{C}$, then the diameter of the resulting particle is small and its height is large. This is shown in Figure 9a. Figure 9b, on the other hand, shows that a flat particle is obtained if both mica and the solution are at $50{ }^{\circ} \mathrm{C}$. The two images shown in Figure 9 are obtained separately by section analysis and then combined. To fit the two shapes into the same figure, the horizontal scale of the flat particle is reduced 10-fol d and the vertical scale is expanded 10fold relative to that of the round one. 


\section{Discussion}

The single PS chain dissolved in $\mathrm{CH}$ is in a state of equilibrium and may be analyzed by using equations from equilibrium thermodynamics and statistical mechanics. However, when a drop of the solution is placed on a mica sheet, the system is strongly out of equilibrium throughout the evaporation stage of the solvent. If the chain remains as a single elastic entity during evaporation and if aggregation of several chains does not take place, one may calculate the deformation ratios of individual chains in going from solution to the dry state. During this process, strong forces act on the single chain. The evaporating liquid exerts a strong compressive force on the chain along the direction perpendicular to the mica surface. The mica opposes this force. The chain is therefore under a strong compression in the direction normal to the mica surface. Flattening in this direction is accompanied by increase of dimensions parallel to the plane of the surface. We denote the deformation perpendicular and parallel to the plane by $\lambda_{\perp}$ and $\lambda_{\|}$, respectively. The deformation $\lambda_{\perp}$ is defined as the final dry dimension perpendicular to the plane at a given temperature divided by the diameter of the coil in solution at the same temperature. Defined in this manner, $\lambda_{\perp}$ denotes the amount of compression experienced by the chain during drying. The parallel or lateral deformation $\lambda_{\|}$is defined as the ratio of the final dry average dimension parallel to the plane at a given temperature divided by the diameter of the coil in solution at the same temperature. This deformation reflects the degree of lateral expansion of the coil as it is deposited on the surface during drying and is similar to the Poisson effect of elasticity.

The dimensions of the chain in solution at any given temperature may be calculated following the method outlined in ref 1 , which we summarize below.

The free energy $\Delta \mathrm{A}$ of the single chain-solvent system is given by

$$
\begin{aligned}
& \Delta \mathrm{A} / \mathrm{RT}=\mathrm{x} \frac{1-\mathrm{v}_{2}}{\mathrm{v}_{2}}\left[\ln \left(1-\mathrm{v}_{2}\right)+\left(\chi_{1}+\frac{\chi_{2}}{2}\right) \mathrm{v}_{2}+\frac{\chi_{2} \mathrm{v}_{2}^{2}}{2}\right]+ \\
& \frac{3}{2}\left[\left(\frac{v_{20}}{v_{2}}\right)^{2 / 3}-1\right]-\ln \left(\frac{v_{20}}{v_{2}}\right)+C
\end{aligned}
$$

Here, $\mathrm{R}$ is the gas constant, $\mathrm{T}$ is the absol ute temperature, and $\mathrm{x}$ is the number of repeat units of the polymer chain whose size is equal to the size of a solvent molecule. (Taking the volume of cyclohexane as 107.7 $\mathrm{cm}^{3} / \mathrm{mol}$ and the density of PS as $1.05 \mathrm{~g} / \mathrm{cm}^{3}$, the value of $x$ is obtained as 17700 for the chains used in the present work.) $v_{2}$ is the volume fraction of polymer in the volume occupied by the single chain; $v_{20}$ is the volume fraction at the $\Theta$ temperature. $C$ is a constant of integration, and $\chi_{1}$ and $\chi_{2}$ are the temperaturedependent components of the $\chi$ parameter, expressed as

$$
\chi=\chi_{1}+\chi_{2} \mathrm{v}_{2}
$$

The expression given by eq 2 assumes that the segments of the chain are uniformly distributed within a sphere of radius proportional to the root-mean-squared endto-end distance of the chain. The volume fraction of polymer at the $\Theta$ temperature is estimated according to ${ }^{1}$

$$
v_{20}=0.84 / x^{1 / 2} \mathrm{P}
$$

where $P$ is given by

$$
\mathrm{P}=\left(\left\langle\mathrm{r}^{2}\right\rangle_{0} / \mathrm{x}\right)^{3 / 2} \mathrm{~V}_{1}^{-1} \mathrm{~N}_{\mathrm{A}}
$$

Here, $V_{1}$ is the molar volume of solvent and $N_{A}$ is the Avogadro number. F or the PS molecule, $\left\langle\mathrm{r}^{2}\right\rangle_{0}$ is obtained by taking the characteristic ratio as 10.2 and the bond length as $1.54 \AA$. Accordingly, the value of $v_{20}$ equates to $3.6 \times 10^{-3}$ for the PS chain of molecular weight $2 \times$ $10^{6} \mathrm{~g} / \mathrm{mol}$.

The $\chi$ parameter given by eq 3 depends strongly on temperature for the system PS- $\mathrm{CH}$. The temperature dependence is calculated according to the equation of state theory for binary mixtures of solvent and polymer. The temperature dependence of the parameters $\chi_{1}$ and $\chi_{2}$ is given in the Appendix of ref 1 and is adopted in the present work.

The single chains in solution form small thermodynamic systems; ther efore, their dimensi ons are expected to exhibit large fluctuations from the mean. The distribution, $W_{x}\left(v_{2}\right)$, of volume fractions is obtained by the use of the expression

$$
W_{x}\left(v_{2}\right)=\frac{\exp (-\Delta A / k T)}{\int_{0}^{1} \exp (-\Delta A / k T) d v_{2}}
$$

The distribution given by eq 6 is for a uniform molecular weight. For the polydisperse system, this distribution is modified as

$$
\mathrm{W}\left(\mathrm{v}_{2}\right)=\sum_{\mathrm{x}} \frac{\mathrm{e}^{-v} v^{\mathrm{x}-1}}{(\mathrm{x}-1) !} \mathrm{W}_{\mathrm{x}}\left(\mathrm{v}_{2}\right)
$$

Here, $W\left(v_{2}\right)$ is the distribution corrected for polydispersity effects, and $v$ is the number-averaged molecular weight. The polydispersity correction here is made by summing the right-hand side of eq 7 over 20 equally spaced stations, centered around $v$ for which the Poisson distribution in the summand is greater than 0.05. The distribution, $W(V)$, of chain volumes is obtained from eq 6 as $^{17}$

$$
W(V)=\frac{V_{d}}{V^{2}} W\left(v_{2}\right)
$$

The dry volume, $V_{d}$, in eq 8 is taken as $3200 \mathrm{~nm}^{3}$ for the chains used in the present experiments.

The distributions of volumes for the four temperatures, $25,35,50$, and $80^{\circ} \mathrm{C}$, are calculated according to eq 8 . The values of the volumes corresponding to the maxima of the distribution curves are presented in the second column of Table 2 . The diameters based on these volumes are given in the third column. Comparison of the dry vol umes given in the second column of Table 1 and corresponding volumes in solution shows that drying induces an average 10-fold volume decrease. A closer inspection of the observed volumes indicates that there is a linear relationship between the dry volumes and the corresponding volumes in solution. This is shown in Figure 10, where the volumes in solution are plotted along the abscissa and the volumes of the dry chains are plotted along the ordinate. The straight line passes close to the origin, denoting that the sets of dry and solution volumes are approximately proportional. 


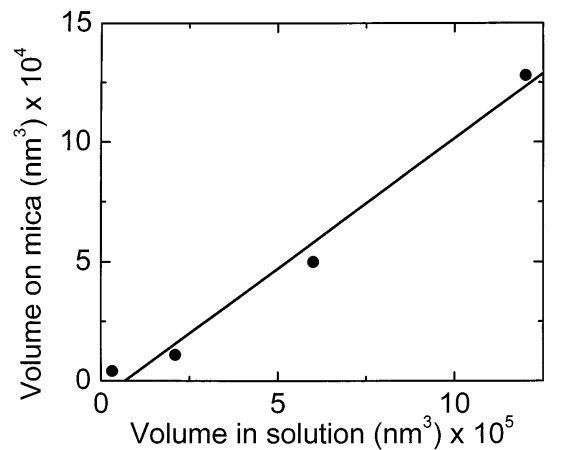

Figure 10. Volume of PS in solution as a function of volume of PS on mica.

The slope of the line is 0.1 , indicating the 10 -fold decrease in volume upon drying. The observed proportionality implies that the distribution of chain sizes transforms affinely during drying. The distribution of dry volumes, $W\left(V_{d}\right)$, may then be obtained from eq 8 according to the expression

$$
\mathrm{W}\left(\mathrm{V}_{\mathrm{d}}\right)=\frac{1}{\lambda} \mathrm{W}\left(\frac{\mathrm{V}}{\lambda}\right)
$$

where $\lambda$ is the volume compression ratio, defined as the volume in solution divided by the volume in the dried state. Results of calculations by taking $\lambda=0.1$ are presented in Figure $11 a-d$ for the four temperatures. The calculated distribution at $25{ }^{\circ} \mathrm{C}$ is significantly sharper than the observed distribution and in this respect does not agree well with experiments. However, the peaks of the calculated and the observed distributions coincide. For all of the higher temperatures, the widths and the peaks of the observed and predicted distributions are in better agreement.

The measured dry diameter and height values are given in columns 4 and 5 of Table 1 . Based on these values, the compression ratio, $\lambda_{\perp}$, perpendicular to the plane and the expansion ratio, $\lambda_{\|}$, in the plane are calculated and shown in columns 4 and 5 of Table 2 . Values obtained for $\lambda_{\perp}$ are much smaller than unity, indicating that the chains are al most fully flattened by the evaporating liquid. Values of $\lambda_{\|}$show, on the other hand, that chain dimensions increase in the plane of the sheet, except for the sample at $35^{\circ} \mathrm{C}$, where the chain dimensions remain the same. At $80^{\circ} \mathrm{C}, \lambda_{\|}=3.72$, indicating that at such high temperatures the chains spread extensively on the surface.

It is to be noted that the size of the chain, $x=17700$, used in the present study is not sufficiently large for the single chain to exhibit a discrete transition from the coil to the globular shape around $35^{\circ} \mathrm{C}$. Such a discrete transition would obtain for chains x larger than 500000 as may be verified from Figure 12 of ref 1 . Despite the much smaller value of the chain length used, the temperature dependence of the present system is significant, as the foregoing results indicate.

The evaporation rate of a drop of $\mathrm{CH}$ on mica is measured in our experiments. The speed of the liquid surface toward the surface of the mica is estimated from these evaporation rates to be on the order of $10^{-4} \mathrm{~cm} / \mathrm{s}$. If the self-diffusion coefficient of the chain segments in solution is assumed to be of the order of $10^{-8} \mathrm{~cm}^{2} / \mathrm{s}$, the lateral expansion speed of the chains becomes of the same order of magnitude as that of the motion of the liquid surface. Apparently, it is this balance between the evaporation rate and the lateral diffusion rate that
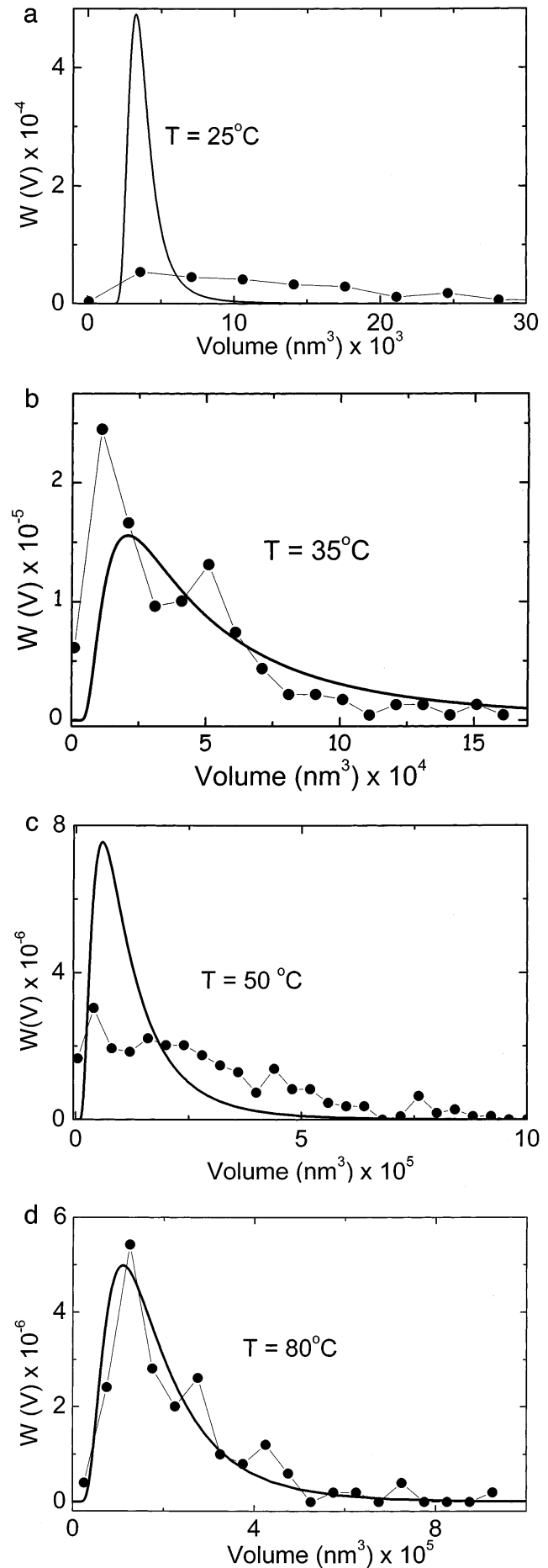

Figure 11. Comparison of cal culated and measured distributions of particle volumes at (a) 25 , (b) 35 , (c) 50 , and (d) $80^{\circ} \mathrm{C}$. The points are from experiments; the line through them is drawn to guide the eye. The solid curve is calculated from theory.

makes the values of $\lambda_{\|}$close to unity. If the evaporation rate were much faster than that of the diffusive motion, then the chain would not have a chance to flatten out, and possibly a mushroom-like structure would obtain. In the other extreme case when the diffusion rate is much faster than the evaporation rate, the chain would fully spread over the surface. At $80^{\circ} \mathrm{C}$, which is very close to the boiling temperature of $\mathrm{CH}$, a larger value of $\lambda_{\|}$may be resulting from a significant enhancement of diffusion rates, in agreement with the present qualitative discussion. 


\section{References and Notes}

(1) Erman, B.; Flory, P. J . Macromolecules 1986, 19, 2342-2353.

(2) Yu, J .; Wang, Z.; Chu, B. Macromolecules 1992, 25, 16181620

(3) Chu, B.; Ying, Q.; Grosberg, A. Yu. Macromolecules 1995, 28 , 180-189.

(4) Chu, B.; Ying, Q. Macromolecules 1996, 29, 1824-1826.

(5) Swislow, G.; Sun, S.; Nishio, I.; Tanaka, T. Phys. Rev. Lett. 1980, 44, 796-798. Sun, S.; Nishio, I.; Swialow, G.; Tanaka, T. J . Chem. Phys. 1980, 73, 5971-5975.

(6) Nierlich, M.; Cotton, J . P.; Farnoux, B. J . Chem. Phys. 1978, 69, 1379-1383.

(7) Vshiskov, S. A.; Safranov, A. P. Macromol. Chem. Phys. 1997, 198, 3015-3023.

(8) Grosberg, A. Yu.; Khokhlov, A. R. Soc. Sci. Rev. A: Phys. 1987, 8, 147-152.

(9) Grosberg, A. Yu.; Nechaev, S. K.; Shakhnovich, E. I. J . Phys. (Paris) 1985, 49, 2095-2103.
(10) de Gennes, P.-G. J . Phys., Lett. 1985, 46, L-689-694.

(11) Qian, R.; Shen, J .; Bei, N.; Zhu, C.; Wang, X. Macromol. Chem. Phys. 1996, 187, 2165-2174.

(12) Festag, R.; Alexandratos, S. D.; Cook, D. K.; J oy, D. C.; Annis, B.; Wunderlich, B. Macromolecules 1997, 30, 6238-6242.

(13) Stange, T. G.; Mathew, R.; Evans, D. F.; Hendricson, W. A. Langmuir 1992, 8, 920-926.

(14) Zhang, L. S.; Manke, C. W.; Ng, K. Y. S. Macromolecules 1995, 28, 7386-7393.

(15) Morris, V. J .; Kirby, A. R.; Gunning, A. P. Atomic Force Microscopy for Biologist, 1st ed.; Imperial College Press: 1999; p 79.

(16) Deegan, R. D.; Olgica, B.; DuPont, T. F.; Huber, G.; Nagel, S. R.; Witten, T. A. Nature (London) 1997, 389, 827-829.

(17) Papoulis, A.; Pillai, S. U. Probability, Random Variables and Stochastic Processes, 4th ed.; McGraw-Hill: New York, 2002; p 131.

MA020676+ 